\title{
Dermoid cysts: Epidemiology and diagnostic approach based on clinical experiences
}

\author{
June Seok Choi ${ }^{1}$, Yong Chan Bae ${ }^{1,2}$, Jae Woo Lee ${ }^{1}$, Gyu Bin Kang ${ }^{1}$ \\ ${ }^{1}$ Department of Plastic and Reconstructive Surgery, Pusan National University School of Medicine, Busan; ${ }^{2}$ Biomedical Research Institute, \\ Pusan National University Hospital, Busan, Korea
}

Background Dermoid cysts are congenital tumors that are benign. Dermoid cysts with intracranial extension can cause serious neurological complications. It is important, therefore, to determine whether a patient has a dermoid cyst when their chief concern at a doctor's visit is a mass in the head or neck area. In this study, we present a literature review of dermoid cysts and an analysis of the authors' experiences, with the goal of providing guidance useful for the diagnosis and treatment of dermoid cysts.

Methods This study retrospectively analyzed the medical records of 62 patients who visited the two medical clinics with which the authors are affiliated. The patients were enrolled between October 2003 and January 2017.

Results Of the 62 patients analyzed in this study, 32 were 0 to 5 years of age (52\%) and 23 were 17 years of age or older (37\%). Forty-seven patients underwent 1 or more imaging study during the process of diagnosis. Thirty-two patients were suspected to have a dermoid cyst. Forty-nine patients were analyzed to determine the depth of the cyst. Bone was seen in 43 patients through imaging tests or during actual surgery, and nine of the 43 had bony problems (21\%).

Conclusions This study found that dermoid cysts were present in many adults, and that a high rate of deep lesions was observed, as well as many cases in which even the bone was affected. These results suggest, therefore, that dermoid cysts should be considered, and medical professionals should actively conduct imaging studies.

Keywords Dermoid cyst / Epidemiology / Diagnosis / Radiology
Correspondence: Yong Chan Bae Department of Plastic and Reconstructive Surgery, Pusan National University School of Medicine, 179 Gudeok-ro, Seo-gu, Busan 49241, Korea Tel: +82-51-240-7273 Fax: +82-51-243-9405 E-mail: baeyc2@hanmail.net

Received: 5 Jan 2018 - Revised: 22 Oct $2018 \bullet$ Accepted: 1 Nov 2018

pISSN: 2234-6163 • elSSN: 2234-6171 • https://doi.org/10.5999/aps.2018.00017• Arch Plast Surg 2018;45:512-516

This work was supported by a clinical research grant from Pusan National University Hospital in 2018.

\section{INTRODUCTION}

Dermoid cysts are known to be benign congenital tumors that develop early in life in ectodermal and mesodermal tissues that are abnormally isolated due to the fusion of the tissues with the bone suture line. Histologically, dermoid cysts are surrounded by keratinizing squamous cells, and contain adnexal structures such as hair follicles, sweat glands, and sebaceous glands. Der- moid cysts with intracranial extension can cause serious neurological complications, such as encephalomeningitis and seizure, when the cyst extends and ruptures, and its contents pass into the subarachnoid space or the cerebral ventricle [1]. It is important, therefore, to determine whether a patient has a dermoid cyst when their chief concern at a doctor's visit is a mass in the head or neck area.

Most dermoid cysts are congenital, and about $70 \%$ are found 
in children 5 years old or younger. They can develop anywhere on the body, but dermoid cysts are most often found in the periorbital lateral eyebrow area (in the head and neck area) [2]. The authors have accumulated considerable experience with dermoid cysts in adults, in contrast to previous observations. Because they are not commonly found in adults, it is possible to overlook dermoid cysts during the diagnostic process.

Previous studies have described dermoid cysts as simply superficial or deep; however, studies exploring this issue are still lacking.

The authors have encountered clinical cases of dermoid cysts that differ from what has been reported in previous studies. Previous studies, furthermore, have not investigated or discussed in detail the depth of the cyst or the appropriate scope of imaging studies. The lack of attention to these details may cause confusion or difficulty in diagnosing dermoid cysts. In this study, we present a literature review of dermoid cysts and an analysis of the authors' experiences, with the goal of providing guidance useful for the diagnosis and treatment of dermoid cysts.

\section{METHODS}

In this study, we retrospectively analyzed the medical records of 62 patients who visited the two medical clinics with which the authors are affiliated. The patients were histologically diagnosed and received surgical treatment for dermoid cysts between October 2003 and January 2017.

We conducted this study after receiving approval from the Institutional Review Board (IRB No. 1810-016-072). Informed written consent was obtained from all patients.

The medical records that were inspected included clinical charts, imaging study examination results, and histopathological results. The analysis included information about the patients' age, sex, clinical manifestation, location of the mass, type of im-

\section{Fig. 1. Age and sex of patients}

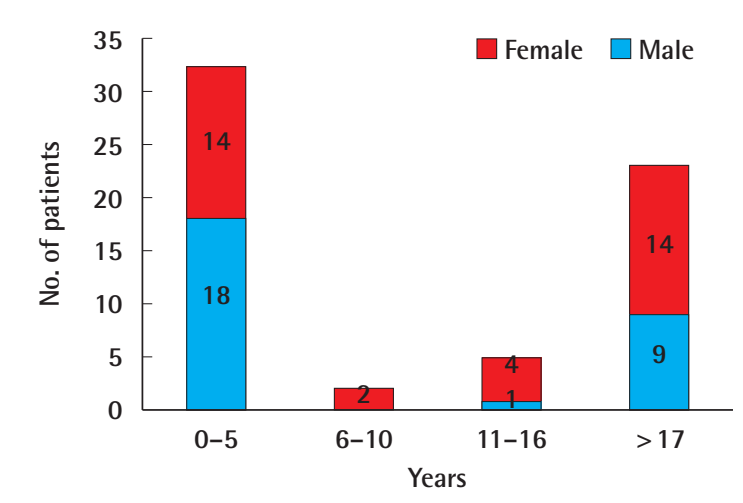

aging studies conducted, and a professional assessment of the results. Further analysis was conducted of the surgical approach, depth of the cyst, and complications. A literature review was conducted, and the authors investigated differences between previous studies and the current study.

\section{RESULTS}

\section{Age and sex}

Of the 62 patients analyzed in this study, 28 were male and 34 were female, with an average age of 14.3 years. Thirty-two patients were 0 to 5 years of age (52\%), two were 6 to 10 years of age (3\%), five were 11 to 16 years of age (8\%), and 23 were 17 years of age or older (37\%) (Fig. 1).

\section{Clinical features}

All patients visited for the assessment of a palpable mass, and 19 of the patients (30\%) had symptoms since birth. Twenty of the 62 patients (32\%) reported a change in size of the mass. Of the 20 who reported a change in size, two had a history of trauma to the lesion.

Three patients showed a local infection (5\%). No symptoms of meningitis or intracranial infection were noted (Table 1).

\section{Location}

The location of the lesions in the 62 patients was categorized into five anatomic sites based on the classification of Pryor et al. [3]. Most of the patients had masses in the periorbital region (52 patients, 84\%) followed by four patients with masses in the ear, three in the nose, one in the occipital region, and two in other areas (Table 1).

\section{Table 1. Location of dermoid cysts and imaging studies}

\begin{tabular}{|lcl|}
\hline Location & $\begin{array}{c}\text { No. of } \\
\text { patients }\end{array}$ & \multicolumn{1}{c|}{$\begin{array}{c}\text { Imaging study } \\
\text { (no. of patients) }\end{array}$} \\
\hline $\begin{array}{l}\text { Periorbital region } \\
\text { Medial brow }\end{array}$ & 52 & \\
Lateral brow & 3 & CT (1) \\
Ear & 49 & CT (33), US (4), MRI (1), US and CT (1), \\
US, CT, and MRI (1)
\end{tabular}




\section{Imaging studies}

Of the 62 patients, 47 underwent 1 or more imaging study: computed tomography (CT) for 37 patients, magnetic resonance imaging (MRI) for three, and ultrasonography (US) for four. Two or more imaging studies were conducted in three patients (Table 1).

Of the 47 patients, only 33 were suspected to have a dermoid cyst. The remaining 14 patients were diagnosed with lipoma (10 patients), an epidermal cyst $(n=2)$, cystic mass $(n=1)$, or an undefined mass $(\mathrm{n}=1)$.

Twenty-six of the 37 patients who were examined through CT were suspected to have a dermoid cyst, as were two of the three who underwent MRI, three of the four who underwent US, and two of the three who underwent 2 or more examinations (Table 2).

Table 2. Radiologic diagnoses

\begin{tabular}{|lccl|}
\hline Image study & Patients & $\begin{array}{c}\text { Dermoid } \\
\text { cyst }\end{array}$ & $\begin{array}{c}\text { Diagnosed as other } \\
\text { than a dermoid cyst } \\
\text { (no. of patients) }\end{array}$ \\
\hline Computed tomography & 37 & 26 & $\begin{array}{c}\text { Lipoma (9), epidermal } \\
\text { cyst (1), cystic mass (1) }\end{array}$ \\
$\begin{array}{l}\text { Magnetic resonance imaging } \\
\text { Ultrasonography }\end{array}$ & 3 & 2 & $\begin{array}{l}\text { Lipoma (1) } \\
\text { Two or more }\end{array}$ \\
\hline
\end{tabular}

\section{Surgical approach}

Surgical removal with a direct approach was performed in all 62 patients. The direct approach was even performed in the patient with an intra-orbital cavity because the patient had only a small bony defect and it did not invade into the dura layer (Fig. 2).

\section{Depth of the cyst}

Records for 49 of the 62 patients allowed an analysis of the depth of the cyst. Nineteen patients had invasion limited to the subcutaneous layer, eight exhibited invasion of the intramuscular layer, 20 showed invasion reaching into the periosteum but with the periosteum intact, and two had invasion extending fully into the periosteum (Table 3).

Bone was seen in 43 patients through imaging studies or during actual surgery, and nine of the patients had bony erosion or a bony defect (21\%) (Fig. 3).

\begin{tabular}{|c|c|}
\hline Depth & No $(\%)$ \\
\hline Subcutaneous layer & 19 (39) \\
\hline Muscular layer & $8(16)$ \\
\hline Periosteum (intact) & $20(41)$ \\
\hline Periosteum (invasion) & $2(4)$ \\
\hline
\end{tabular}

\section{Fig. 2. Case}

(A) Preoperative photo and (B) postoperative photo. (C, D) Preoperative magnetic resonance imaging examination.
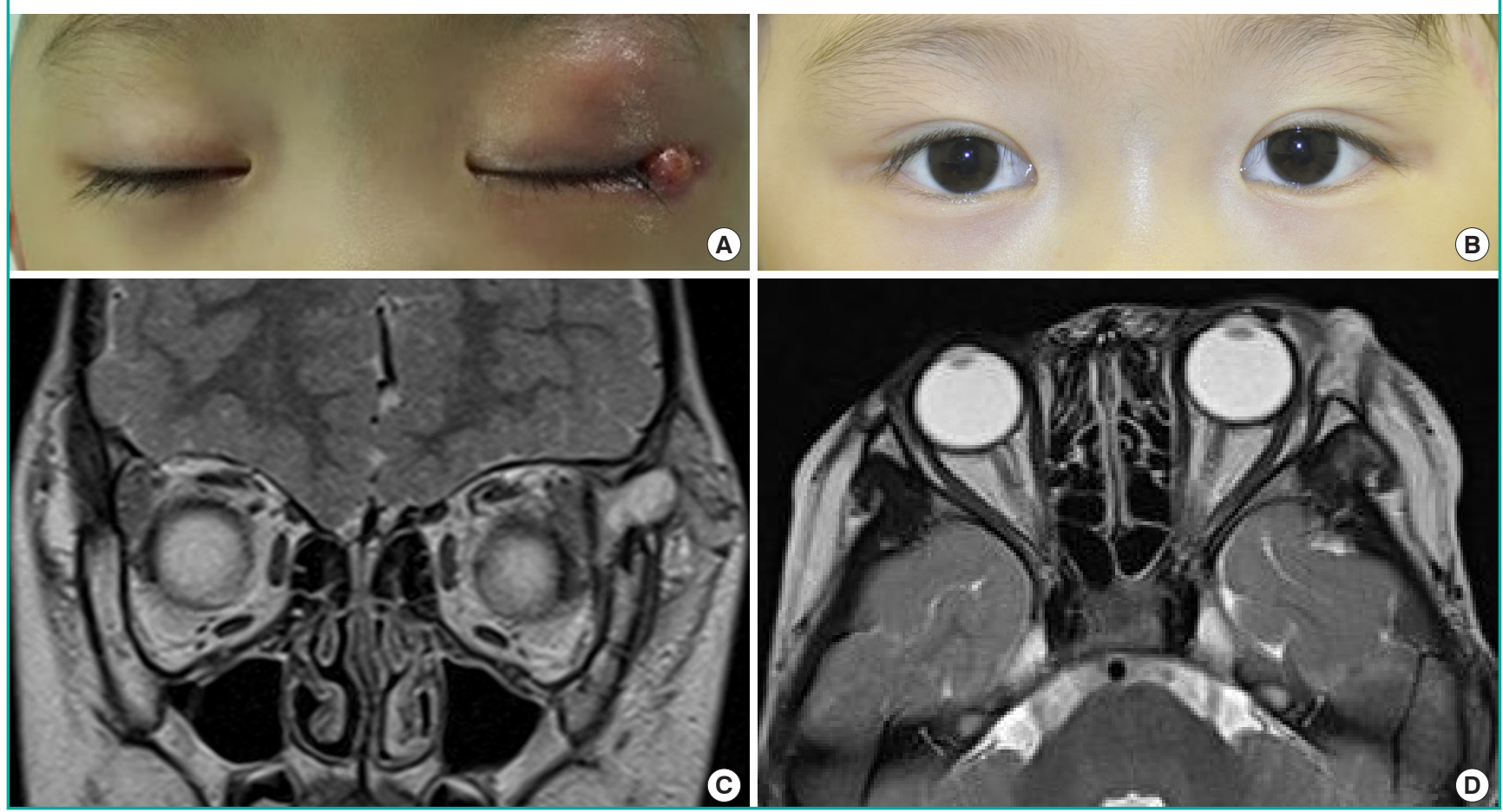
Fig 3. Case of bone erosion

A patient who had bony erosion (red arrow): computed tomography finding. (A) Coronal and (B) axial.

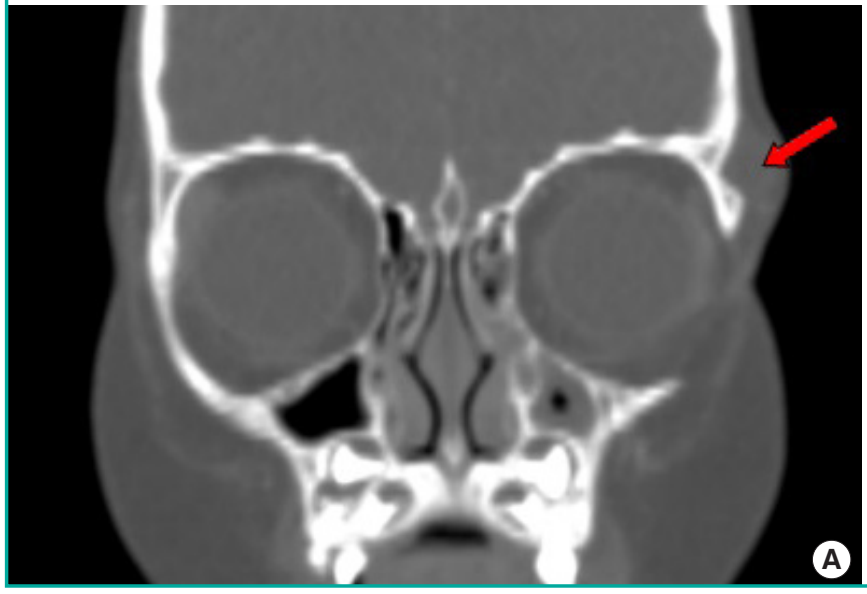

\section{Complications}

No cases of recurrence were observed in the patients who underwent surgery, and other than three patients who claimed that they had irregular contouring or scarring, no other serious complications were noted.

\section{DISCUSSION}

Most dermoid cysts are congenital, and it is known that approximately $70 \%$ of cases are found in children 5 years old or younger [2]. However, this study found that only $30 \%$ of the patients investigated had congenital symptoms, and the rate of dermoid cysts in children 5 years old or younger was lower (52\%) than has been reported elsewhere. In contrast, this study found many patients 17 years of age or older (23 patients, 37\%). These results suggest, therefore, that dermoid cysts in adults should not be overlooked, and should be taken into consideration when diagnosing patients.

For the first time in medical history in 1937, New and Erich [2] categorized congenital inclusion dermoid cysts in the head and neck area into four groups: the periorbital region (group 1), the nose (group 2), the submental region (group 3), and the midventral and mid-dorsal fusion area of the suprasternal, thyroidal, and suboccipital region (group 4).

After the initial categories were established, studies found dermoid cysts in areas that were not included in the first categorization, and Pryor et al. [3] designated such areas as "head not neck." Dermoid cysts in this new group develop slowly and rarely show extension; therefore, Pryor suggested that minimal imaging studies sufficed for diagnosing patients. However, according to our literature review, dermoid cysts that develop in the scalp or ear regions have been reported to exhibit intracranial

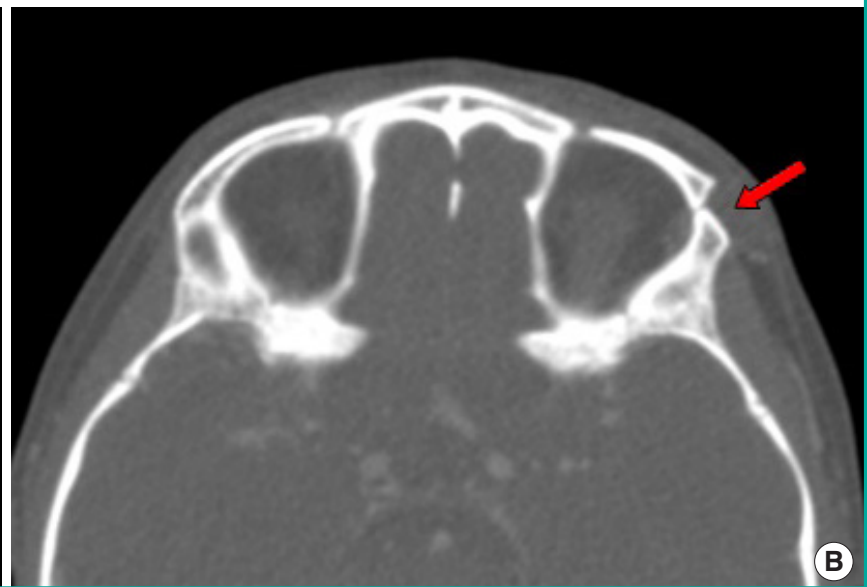

extension $[4,5]$, suggesting that caution is important when treating these areas. The authors, therefore, found it necessary to supplement the prior categorizations of New and Erich [2] and Pryor et al. [3] and developed a classification that includes the scalp and ear regions in which intracranial extension is possible (Table 1). In agreement with previous research, this study also found that most cases of dermoid cysts occurred in the periorbital region, in 52 of 62 patients (84\%) [3]. In our study, there were no cases of intracranial dermoid cysts. According to a previous study, intracranial cases are rare, and there is a possibility of rupture when they are operated on without a radiological examination. Since rupture in such cases can be life-threatening for the patient, we propose that radiological examinations should be recommended [1].

Imaging studies can be conducted based on the patient's health care plan or the preference of the surgeon. In previous studies, imaging studies were recommended because dermoid cysts in the medial brow sometimes show intracranial extension, but imaging studies have not been considered mandatory because dermoid cysts that develop in the lateral brow region are even rarer [6,7]. However, the authors discovered a suspicious intracranial extension in the lateral canthal region. As suggested by previous research, since intracranial extension can be observed in the medial canthal region, nasal region, scalp, and periauricular region, imaging studies must be performed in the lateral canthal region. Furthermore, if inflammation or infection in the lateral canthal region repeats, a more aggressive imaging study should be conducted. CT was the most commonly used imaging modality for the patients in this study, and it did not show a difference in the diagnoses compared to other methods (Table 2). CT can show the location and content of the mass, as well as its relationship to the surrounding structures and their 
condition, while also allowing the physician to confirm intracranial extension indirectly $[8,9]$. The authors, therefore, view CT as the most useful imaging modality for patients in whom a dermal cyst is suspected. However, an imaging-based diagnosis does not always correspond to the actual diagnosis; therefore, it should be used as a diagnostic supplement.

Dermoid cyst treatment can be performed through a direct approach or endoscopic approach, depending on the surgeon's preference. Craniotomy can accompany the treatment if intracranial extension is found [10]. The authors performed a direct approach in all patients. Only one patient showed serious problems with scarring. Given these results, if appropriate scar management is part of the follow-up, excision through a direct approach is preferable. Five of the patients who visited the authors' clinics came in because the cyst had not been completely removed in a prior procedure at a different clinic or because the cyst had recurred after only aspiration was performed. It can be seen, therefore, that the complete removal of the mass is important, regardless of the method.

The layers containing the dermoid cysts were analyzed in 49 of the patients in this study. Previous studies have not investigated the depth of the lesions, and the authors believe that this analysis can provide a standard for estimating the depth during surgery. Twenty patients had deep lesions in the superior periosteum (41\%), and two patients had deep lesions in the inferior periosteum (4\%). Among the 43 patients that showed bone in the CT results prior to surgery or in whom bone was found during surgery, nine patients had bony erosion or a bony defect, corresponding to a considerable rate of $21 \%$.

There are several limitations to our study, First, the number of cases is too small to generalize our results. Another limitation is that our study did not include a true case of intracranial extension. However, it did include a case of a cyst that almost invaded the skull, which can be dangerous enough in its own right.

This study found numerous cases of dermoid cysts in adults, in the periorbital region, a higher rate of deep lesions, and cases that even affected the bone. These results suggest, therefore, that dermoid cysts should be taken into consideration regardless of age (especially in adults) and the location (especially in the periorbital region) of the mass, and that the possibility of intracranial extension must be taken into account. Additionally, medical professionals should actively conduct imaging studies such as axial CT scans to determine whether a mass is a dermoid cyst and to diagnose it properly after assessing its correlations with the clinical diagnosis. Finally, we should consider the likelihood of deep-positioned cysts and perform appropriate dissection in such cases.

\section{NOTES}

\section{Conflict of interest}

No potential conflict of interest relevant to this article was reported.

\section{Ethical approval}

The study was approved by the Institutional Review Board of Pusan National University Hospital (IRB No. 1810-016-072) and performed in accordance with the principles of the Declaration of Helsinki. Written informed consents were obtained.

\section{Patient consent}

The patient provided written informed consent for the publication and the use of her images.

\section{REFERENCES}

1. Ray MJ, Barnett DW, Snipes GJ, et al. Ruptured intracranial dermoid cyst. Proc (Bayl Univ Med Cent) 2012;25:23-5.

2. New GB, Erich JB. Dermoid cysts of the head and neck. Surg Gynecol Obstet 1937;65:48-55.

3. Pryor SG, Lewis JE, Weaver AL, et al. Pediatric dermoid cysts of the head and neck. Otolaryngol Head Neck Surg 2005; 132:938-42.

4. Kim BY, Park JS, Kang MK, et al. A case of dermoid cyst in temporal bone. Korean J Otorhinolaryngol-Head Neck Surg 2007;50:1043-6.

5. Crawford R. Dermoid cyst of the scalp: intracranial extension.J Pediatr Surg 1990;25:294-5.

6. Sathananthan N, Moseley IF, Rose GE, et al. The frequency and clinical significance of bone involvement in outer canthus dermoid cysts. Br J Ophthalmol 1993;77:789-94.

7. Richards AM. Key notes on plastic surgery. Oxford: Blackwell Publishing; 2002.

8. Mann GS, Gupta A, Cochrane DD, et al. Occipital dermoid cyst associated with dermal sinus and cerebellar abscesses. Can J Neurol Sci 2009;36:487-90.

9. Posnick JC, Bortoluzzi P, Armstrong DC, et al. Intracranial nasal dermoid sinus cysts: computed tomographic scan findings and surgical results. Plast Reconstr Surg 1994;93: 745-54.

10. Guerrissi JO. Endoscopic excision of frontozygomatic dermoid cysts. J Craniofac Surg 2004;15:618-22. 